\title{
MIGRATION ANADROME ESTUARIENNE DES GÉNITEURS DE GRANDE ALOSE ALOSA ALOSA, ALLURE DU PHÉNOMĖNE ET INFLUENCE DU RYTHME DES MARÉES.
}

\author{
E. ROCHARD \\ Cemagref Unité ressources aquatiques continentales, 33610 CESTAS Cedex, France. \\ E-mail : eric.rochard@ cemagref.fr
}

\section{RÉSUMÉ}

Pour accéder à leurs zones de frayères les géniteurs de grande alose Alosa alosa L. 1758, traversent un estuaire où, en fonction du rythme des marées, les conditions hydrauliques sont tantôt favorables tantôt défavorables à leurs déplacements. Dans ce travail nous nous sommes intéressés à la migration des géniteurs de grande alose lors de leur traversée de l'estuaire de la Gironde (France). Pour cela nous nous sommes basés sur des données de captures par unité d'effort (CPUE) quotidiennes obtenues auprès de pêcheurs commerciaux sur deux secteurs de l'estuaire lors des saisons de migrations 1985-1988. Ces séries chronologiques de CPUE ont été analysées par des méthodes de traitement du signal : extraction des tendances saisonnières et analyse de la variabilité sur les séries résiduelles. Nous avons ainsi mis en évidence une tendance du phénomène uni ou bimodale selon les années, ceci en liaison avec l'évolution de la température de l'estuaire. Le décalage des pics de migration, entre les zones aval et amont a permis d'estimer la vitesse de migration des aloses sur ce secteur à $17-23 \mathrm{~km} . \mathrm{j}^{-1}$. Les séries résiduelles montrent des phénomènes cycliques, significatifs et d'une périodicité de 1416 jours et 28 jours dans le secteur aval, mais moins nets et non significatifs à l'amont. Sur le secteur aval il existe une corrélation avec retard entre la série résiduelle et l'importance de la marée, par contre ce décalage varie d'une année à l'autre de 0 à 6 jours. Ceci pourrait être la conséquence du passage des migrants à travers les champs des courants estuariens. Dans le secteur amont, la corrélation avec les marées est 3 fois plus faible en intensité et nettement moins régulière que dans le secteur aval. Ceci pourrait provenir de l'atténuation progressive de l'influence de la marée et de l'émergence au sein du phénomène migratoire d'un rythme d'une périodicité de 7 jours induit par le fonctionnement de la pêcherie. rythme.

Mots-clés : grande alose, Alosa alosa, migration, estuaire, marée, température,

\section{ANADROMOUS ESTUARINE MIGRATION OF ADULT ALLIS SHAD ALOSA ALOSA, SHAPE OF THE PHENOMENON AND INFLUENCE OF THE TIDAL CYCLE.}

\begin{abstract}
To reach their spawning grounds, adults of Allis shad Alosa alosa L. 1758 have to go through an estuary. In this area, according to the tidal rhythm, hydraulics conditions are sometimes favorable and some times unfavorable to their upstream migration. In this work we focus our attention on the Allis shad anadromous migration during their crossing of the


Gironde estuary (France). We based our analysis on daily catch by unit of effort (CPUE) data from commercial fishermen. They have been collected in two sectors of the estuary during migration seasons 1985-1988. These CPUE time series which represents the evolution of the migration have been analyzed with signal analysis methods : trends extraction, residuals variability analysis. We established that the shape of the phenomenon is uni or bimodal, the trend depends of the temperature evolution during the migration season. The time lag between peaks in the two sectors enable us to assess the migration speed of the shad in estuary : 17-23 km.d ${ }^{-1}$. Residuals time series exhibit cyclic components, in the downstream sector they are significant and periods are : 14-16 d and $28 \mathrm{~d}$ in the upstream sector their shape is less regular and they are not significant. On the lower sector the lag cross correlation between residuals and tide magnitude is significant, the time lag varies from year between 0 and $6 \mathrm{~d}$. This could come from the crossing by the migrants of estuarine tidal current fields. In the upstream sector correlation with tides is 3 times lower in intensity and less regular. This could result of the progressive smoothing of the tidal cycle and of the appraisal in the migration phenomenon of a $7 \mathrm{~d}$ periodicity which could be linked to the fisheries working.

Key-words : allis shad, Alosa alosa, migration, estuary, tide, temperature, rhythm.

\section{INTRODUCTION}

Les géniteurs des populations atlantiques de grande alose Alosa alosa doivent nécessairement traverser un estuaire pour accéder à leurs zones de frayères, situées dans la partie moyenne des fleuves (BAGLINIĖRE et ELIE, 2000). Cette phase de leur migration de reproduction se déroule dans un environnement de transition, dont certaines caractéristiques hydrodynamiques et physico-chimiques fluctuent plus fortement et plus rapidement que dans les milieux océanique ou fluvial.

Les informations concernant la phase marine du cycle de vie des aloses sont rares (voir LEGGETT et WHITNEY, 1972 ; NEVES et DEPRES, 1979 et PARKER, 1992 pour A. sapidissima ou TAVERNY et al., 2000 et TAVERNY et ELIE, 2001, pour A. alosa et $A$. fallax) et ne permettent pas de préciser l'initiation de la migration anadrome de reproduction. Par contre, la phase fluviale à fait l'objet de nombreuses études visant à identifier l'influence de la température ou du débit fluvial. ROULE (1924) en examinant le partage des $A$. alosa à des confluences concluait ainsi à l'existence d'un thermotropisme positif, résultat que ne retrouve toutefois pas MARTIN VANDEMBULCKE (1999). II est généralement admis l'existence d'une température seuil en dessous de laquelle on observe peu d'activité migratoire (voir TALBOT, 1954 ; MASSMAN et PACHECO, 1957 ; LEGGETT et WHITNEY 1972 pour $A$. sapidissima et DAUTREY et LARTIGUE, 1983 ; MENNESON-BOISNEAU 1990 et MENNESON-BOISNEAU et al., 2000 pour A. alosa). Ce seuil qui pourrait se situer autour de $11^{\circ} \mathrm{C}$ pour la grande alose en France (MENNESONBOISNEAU et al., 2000) serait variable selon les rivières mais également selon les conditions climatiques (BELAUD et LABAT, 1985 ; STEINBACH et al., 1986). La vitesse de nage, très variable selon les individus, est partiellement fonction de la vitesse du courant et diminue sensiblement au dessus de $20^{\circ} \mathrm{C}$ (KATZ, 1986), ceci pourrait expliquer le lien établi par certains auteurs (ex. DAUTREY et LARTIGUE, 1983 ; MENNESONBOISNEAU, 1990 ; SABATIÉ, 1993 ; MENNESON-BOISNEAU et al., 2000) entre la progression des aloses en rivière et la température de l'eau. L'action du débit fluvial sur la migration est moins nette (TALBOT, 1954 ; LEGGETT et WHITNEY, 1972 ; DAUTREY et LARTIGUE, 1983 ; PROUZET et al., 1994) et d'une importance variable selon le moment au sein de la migration. II pourrait agir comme modulateur secondaire lorsque la température est favorable (RICHKUS, 1974 ; PROUZET et al., 1994).

Les estuaires atlantiques sont soumis à une hydrologie particulière qui dépend de la morphologie du lit, du débit fluvial et de l'intrusion marine. Cette dernière est régie par les 
marées qui, sur les côtes européennes, sont de type semi-diurnes. En France l'amplitude de cette oscillation est quantifiée par le "coefficient de marée ", qui varie de 20 (plus faible amplitude) à 120 (plus forte amplitude). Dans les estuaires macrotidaux, en raison de la faible profondeur et du débit fluvial, la durée de la marée descendante (jusant) est prépondérante par rapport à la durée de la marée montante (flot) et augmente vers l'amont. Cette configuration conduit à la création de courants puissants, alternativement vers l'amont et vers l'aval.

Pour franchir ces milieux à conditions hydrodynamiques alternées, certaines espèces de poissons ont développé des stratégies tenant compte de cette rythmicité. C'est ainsi que l'utilisation sélective des courants de marée pour pénétrer dans les estuaires a été mis en évidence chez certaines espèces migratrices comme les anguilles Anguilla anguilla et Anguilla rostrata, le flet Platichthys flesus ou même des espèces marines utilisant les estuaires au cours de leur cycle vital comme la sole Solea solea ou la plie Pleuronectes platessa (voir MAC CLEAVE et WIPPELHAUSER, 1987, pour une revue plus exhaustive de cet aspect). Ces stratégies visent à minimiser les dépenses énergétiques pour la traversée de l'estuaire en utilisant les marées montantes pour progresser vers l'amont et en esquivant le jusant.

Hormis CASTELNAUD (1978) qui a rapporté l'importance accordée par les pêcheurs aux coefficients de marée, MENNESSON-BOISNEAU (1990) qui a décrit les grandes lignes du phénomène dans la partie amont de l'estuaire de la Loire et PROUZET et al. (1994) qui ont mené une analyse globale de la migration sur l'Adour, la migration estuarienne de la grande alose $A$. alosa n'a pas fait l'objet d'analyse spécifique. Dans ce travail nous avons pour objectif de caractériser la migration des géniteurs de grande alose Alosa alosa lors de leur traversée de l'estuaire de la Gironde en examinant particulièrement les composantes cycliques pouvant être reliées aux marées de façon à en tirer des conclusions écologiques quant à la stratégie de migration des aloses au travers des estuaires.

\section{MATÉRIEL ET MÉTHODES}

\section{Zone d'étude}

Dans le bassin versant Gironde-Garonne-Dordogne, la zone soumise à l'influence de la marée dynamique s'étend sur $160 \mathrm{~km}$ (Figure 1). Le marnage atteint $4,5 \mathrm{~m}$ à l'embouchure et plus de 5,0 $\mathrm{m}$ à Bordeaux (90 km de l'embouchure). Le module du débit fluvial (Garonne + Dordogne) est de $989 \mathrm{~m}^{3} . \mathrm{s}^{-1}$ (ALLEN, 1972) ; les eaux douces s'écoulent préférentiellement le long de la rive droite et l'intrusion marine s'effectue plutôt le long de la

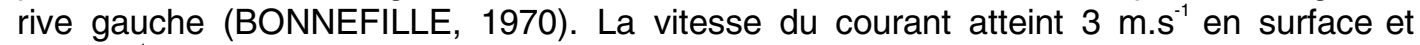
$1,5 \mathrm{~m}^{-1} \mathrm{~s}^{-1}$ à $1 \mathrm{~m}$ du fond lors des marées de vives eaux (MAURICE, 1994). Les volumes d'eau mis en jeu dans cet estuaire lors d'un cycle de marée montrent clairement l'influence prépondérante de l'intrusion marine $\left(1,1\right.$ à $\left.2,0.10^{9} \mathrm{~m}^{3}\right)$ sur le débit fluvial (en moyenne $0,04.10^{9} \mathrm{~m}^{3}$ ) (BONNEFILLE, 1970). La turbidité varie de $1 \mathrm{~g} . \mathrm{l}^{-1}$ près de la surface à $10 \mathrm{~g} . \mathrm{l}^{-1}$ près du fond (LATOUCHE et JOUANNEAU, 1994). Le bouchon vaseux typique des estuaires atlantiques est présent, mais on n'a pas noté jusqu'alors d'anoxie comme c'est le cas par exemple dans l'estuaire de la Loire (SAURIAU et al., 1991). La température suit un léger gradient $\left(1^{\circ} \mathrm{C}\right)$ qui s'inverse deux fois par an, au printemps et en automne. Les eaux côtières sont plus chaudes que les eaux fluviales en hiver, alors que c'est le contraire en été (C.N.EX.O. 1977). La salinité suit également un gradient longitudinal, très variable en fonction de la force de la marée et du débit fluvial.

Les géniteurs d'Alosa alosa effectuent leur migration de reproduction à travers l'estuaire de la Gironde entre avril et juin ; d'après TAVERNY (1991), $95 \%$ des individus n'accomplissent ce trajet qu'une seule fois. Durant leur traversée de l'estuaire les aloses 
font l'objet d'une pêche commerciale à l'aide de filets tramails dérivants (CASTELNAUD, 1978), les pêcheurs les capturant selon les secteurs de l'estuaire à des moments variables du cycle tidal (CASTELNAUD, comm. pers.).

Nos données proviennent de deux secteurs (Figure 1) : l'un situé à l'amont en zone dulçaquicole sur la Garonne est soumis seulement à la marée dynamique (variation de niveau et inversement du sens de l'écoulement des eaux), c'est le secteur principal de pêche de cette espèce ; l'autre situé à l'aval, en zone saumâtre sur la Gironde est soumis à la marée dynamique et à des variations de salinité mais la pêche de l'alose y est moins intense (ROCHARD, 1992).

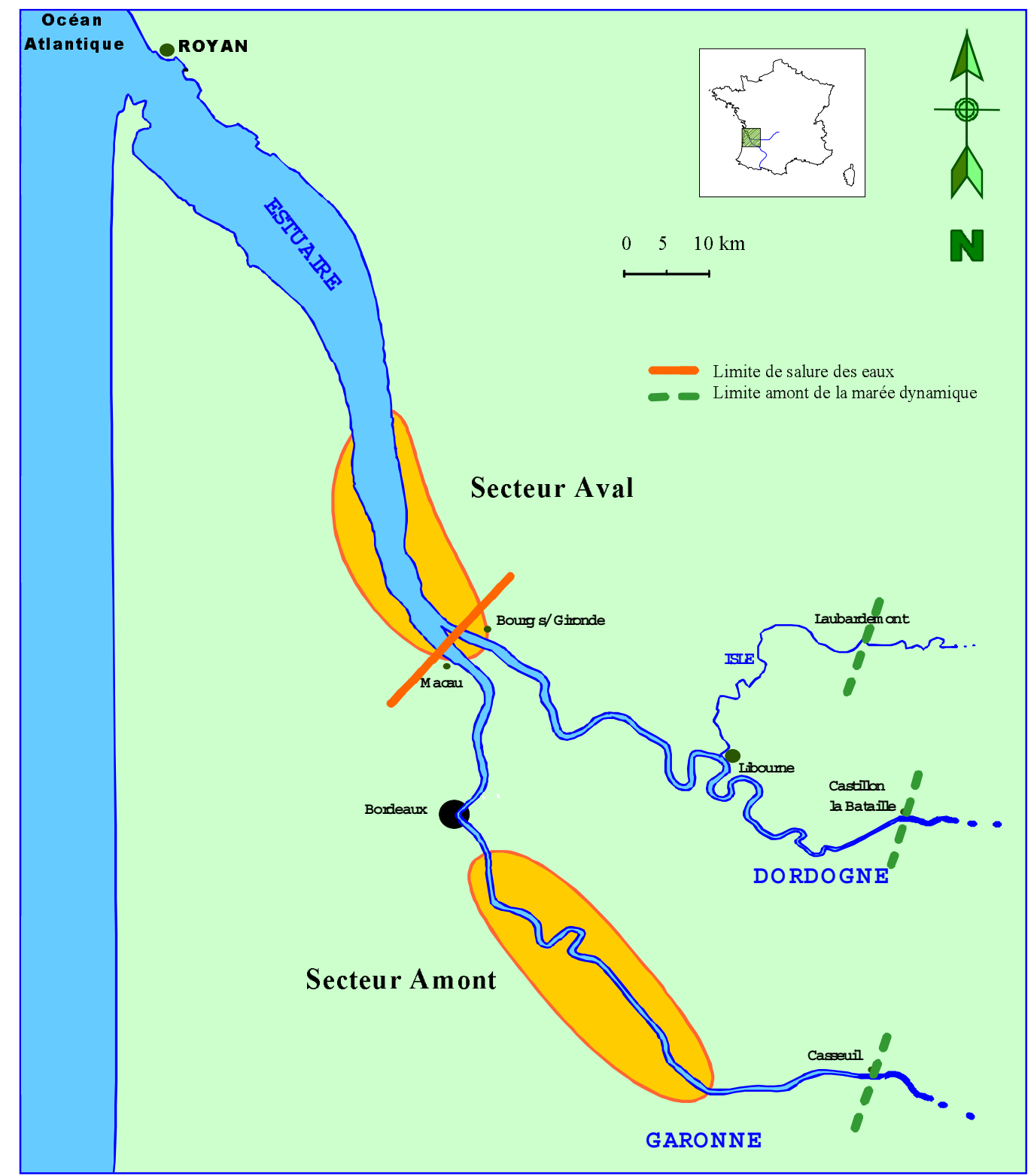

\section{Figure 1}

Localisation des deux secteurs d'étude dans l'estuaire de la Gironde.

\section{Figure 1}

Localization of the study sectors in the Gironde estuary. 
Le débit fluvial (Garonne + Dordogne) nous a été communiqué par le Port Autonome de Bordeaux, les températures de l'eau de l'estuaire ont été mesurées à la prise d'eau du Centre Nucléaire de Production d'électricité du Blayais par Electricité De France.

\section{Méthode retenue}

La migration des géniteurs de grande alose (i.e. la variation de leur abondance locale au cours du temps) a été estimée dans chacun des secteurs retenus par les captures par unité d'effort quotidiennes (CPUE) de pêcheurs commerciaux au filet tramail. Ces données proviennent d'un réseau mis en place au début des années 80 (voir ROCHARD, 1992 ou CASTELNAUD et al., 2001, pour plus de précisions). Ces pêcheurs nous ont indiqué de façon volontaire, pour chaque date, le lieu de pêche et le nombre d'aloses capturées. Pour cette analyse nous avons préféré sélectionner des pêcheurs recherchant la grande alose durant toute la saison de migration dans un même secteur et non pas seulement au moment du pic, ceci limite naturellement le nombre de séries utilisables. Ces données ont permis le calcul quotidien de CPUE moyennes pour chacun des deux secteurs et constituent nos séries brutes. Les données utilisées proviennent pour le secteur aval des saisons de migration 1985 (4 pêcheurs), 1986 (5), 1987 (2), et 1988 (2) ; et pour le secteur amont des saisons 1985 (3 pêcheurs), 1986 (3), 1987 (4) et $1988(6)$.

Nous avons considéré l'évolution dans le temps de l'abondance des aloses comme la superposition de trois processus : la tendance de la migration, des phénomènes cycliques modulateurs que l'on va chercher à identifier et des épiphénomènes perturbateurs accidentels qui ne seront pas considérés ici. La tendance est un processus de basse fréquence (FORTIER et al., 1978 ; LEGENDRE et LEGENDRE, 1979) que nous considérons comme un phénomène cyclique de période annuelle dépendant de l'écologie des populations mais susceptible d'être influencé par les conditions environnementales précédant ou accompagnant le phénomène. Une approche du même type a été utilisée par SAILA et al., (1972) pour mettre en évidence les rythmes d'activités de A. pseudoharengus en rivière.

La tendance de la migration $\left(Y_{j}\right)$ est extraite à partir de la série brute $\left(\mathrm{y}_{\mathrm{j}}\right)$ en filtrant tous les phénomènes d'une périodicité inférieure ou égale à 29 j (mois lunaire) grâce à une moyenne mobile pondérée d'ordre 29. Le facteur de pondération $w_{i}$ est symétrique par rapport à j et la somme des facteurs de pondération est égale à 1 .

$$
\begin{aligned}
Y_{j} & =\sum_{i} y_{(j+i))} w_{i} \quad[-14, i,+14] \\
w_{i} & =\frac{(14+i)-|i|}{\sum_{i}(14+i)-|i|}
\end{aligned}
$$

La tendance est considérée comme la résultante de une ou plusieurs vagues de migrations gaussiennes, elle a été définie en fonction du temps.

Par soustraction de la tendance à la série brute on obtient une série résiduelle. Les rythmes éventuels au sein des séries résiduelles ont été identifiés et caractérisés en calculant leurs autocovariances $S_{y y}(k)$ et leurs coefficients d'autocorrélation $\left(r_{y y k}\right)$ (LEGENDRE et LEGENDRE, 1979). Ce coefficient d'autocorrélation est une mesure de la dépendance entre une observation effectuée en j et en ( $\mathrm{j}+\mathrm{k})$ (FORTIER et al., 1978). La représentation de $r_{y y}$ en fonction de $k$, appelé corrélogramme permet de caractériser la série et de déterminer son rythme interne. Son intervalle de confiance a été évalué selon 
la formule de COX et LEWIS (1966). L'autocorrélation est statistiquement différente de 0 en dehors de l'intervalle suivant :

$$
-\left[\frac{t}{\sqrt{(p-1-k)}}\right]<r_{y y}<\left[\frac{t}{\sqrt{(p-1-k)}}\right] \quad(p \leq 0,05)
$$

$\mathrm{t}:$ valeur du $\mathrm{t}$ de STUDENT pour la série brute

Nous avons alors testé le rôle possible des marées comme synchroniseurs de la migration de l'alose en calculant les corrélations croisées avec retard entre les séries résiduelles et les séries chronologiques des coefficients de marée.

\section{RÉSULTATS}

\section{Tendance du phénomène}

Les tendances migratoires sont identiques dans les deux secteurs où seuls le positionnement temporel et l'intensité de la migration varient. Elles sont : nettement bimodales en 1985, légèrement bimodales en 1987 et 1988 et unimodales en 1986 (Figure 2) ; lorsqu'il y a deux modes leur importance relative est variable. Nous avons choisi d'illustrer ce volet avec des figures concernant le secteur amont (voir ROCHARD, 1992 pour des illustrations concernant le secteur aval). Les modes se situent lors de marées de mortes eaux (coefficient de marée $<52$ ), le premier aux alentours du 25 avril et le second 24-28 jours plus tard. En 1986 le mode unique se situe le 15 mai soit juste au centre de la période principale de migration (15 avril-15 juin).
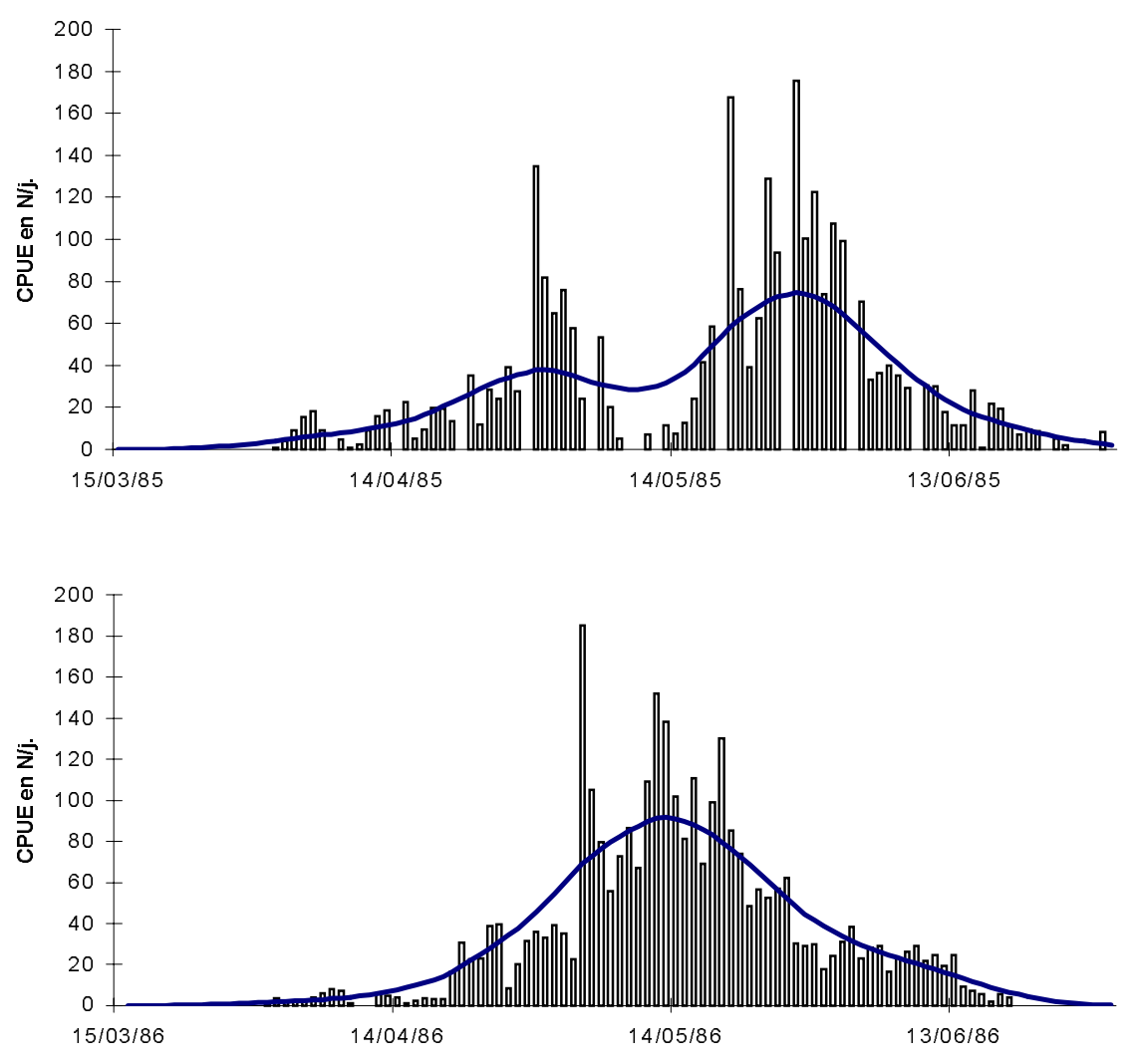

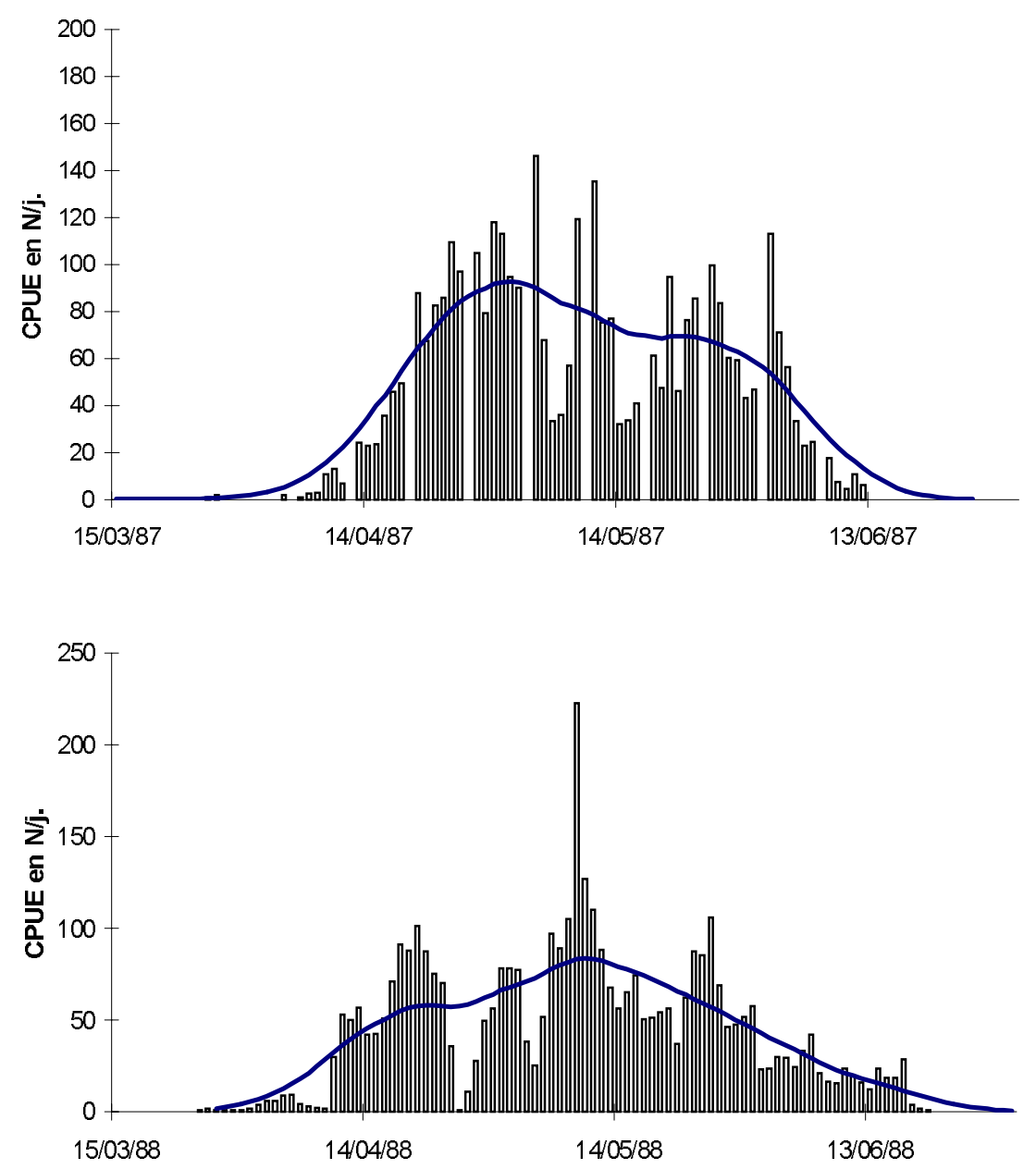

\section{Figure 2}

Séries brutes des CPUE (histogramme) et tendances de la migration (courbe) dans le secteur amont pour les saisons 1985-1988.

\section{Figure 2}

Time series of CPUE (histogram) and trends of the migration (line) in the upstream sector for seasons 1985-1988.

Les quatre courbes de tendances sont suffisamment proches pour les assimiler à une tendance unimodale (ex. 1986) pouvant se transformer suite à une contrainte externe en légèrement bimodale (ex. 1987, 1988) voire franchement bimodale (ex. 1985).

Au cours des quatre années d'observations, les migrations se sont déroulées entre 7,5 et $24^{\circ} \mathrm{C}$ (Tableau I) avec $90 \%$ des migrants qui ont transité, selon les années, à des températures inférieures à $17,5-20^{\circ} \mathrm{C}$. Une seule fois (en 1985) la température a franchement et durablement diminué au milieu de la saison de migration (suite à une crue) (Figure 3). On remarque que ceci correspond à la seule saison présentant une tendance nettement bimodale. Une autre baisse de température a été observée en 1986 (là encore suite à une crue) mais en tout début de migration ce qui n'a pas entraîné de répercussion sur la forme de la tendance. Une forte crue accompagnée d'une baisse très légère de la température a eu lieu au premier tiers de la saison de migration en 1988, l'influence sur la forme de la tendance apparaît très légère. 


\section{Tableau I}

Gamme de température durant laquelle la migration a été observée en estuaire $\left({ }^{\circ} \mathrm{C}\right)$, températures correspondant à $50 \%$ et à $90 \%$ de la migration (tendance).

\section{Table I}

Intervals of temperature within migration was observed in the estuary $\left({ }^{\circ} \mathrm{C}\right)$, temperatures reached respectively at $50 \%$ and $90 \%$ of the migration (trend).

\begin{tabular}{lccc}
\hline & Min. - Max. & 50 \% migration & 90 \% migration \\
\hline 1985 & $9,0-21,0$ & $<15,0$ & $<20,0$ \\
1986 & $7,5-24,0$ & $<16,0$ & $<19,5$ \\
1987 & $9,0-22,0$ & $<16,0$ & $<18,0$ \\
1988 & $10,0-21,5$ & $<16,0$ & $<17,5$ \\
\hline
\end{tabular}
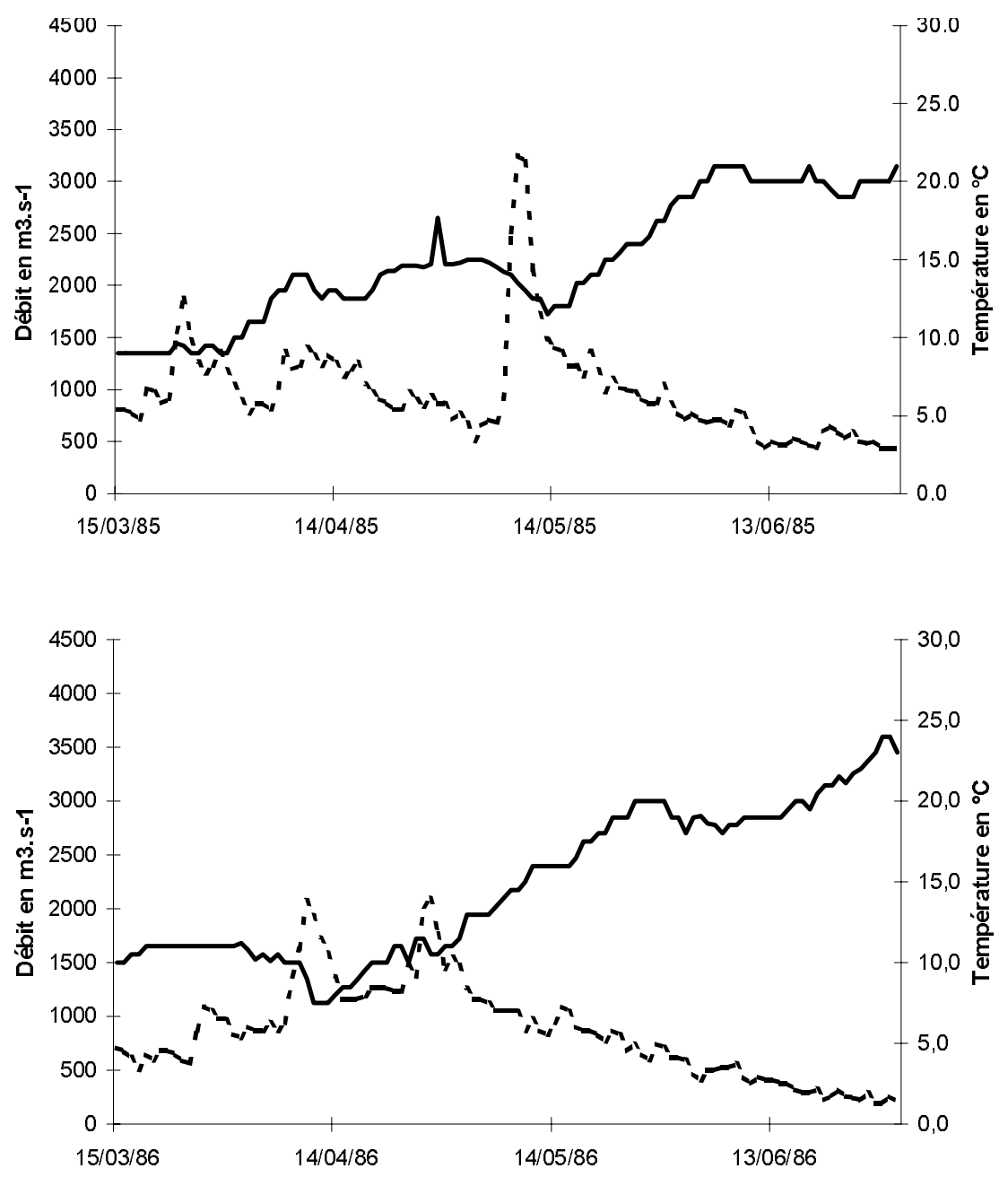

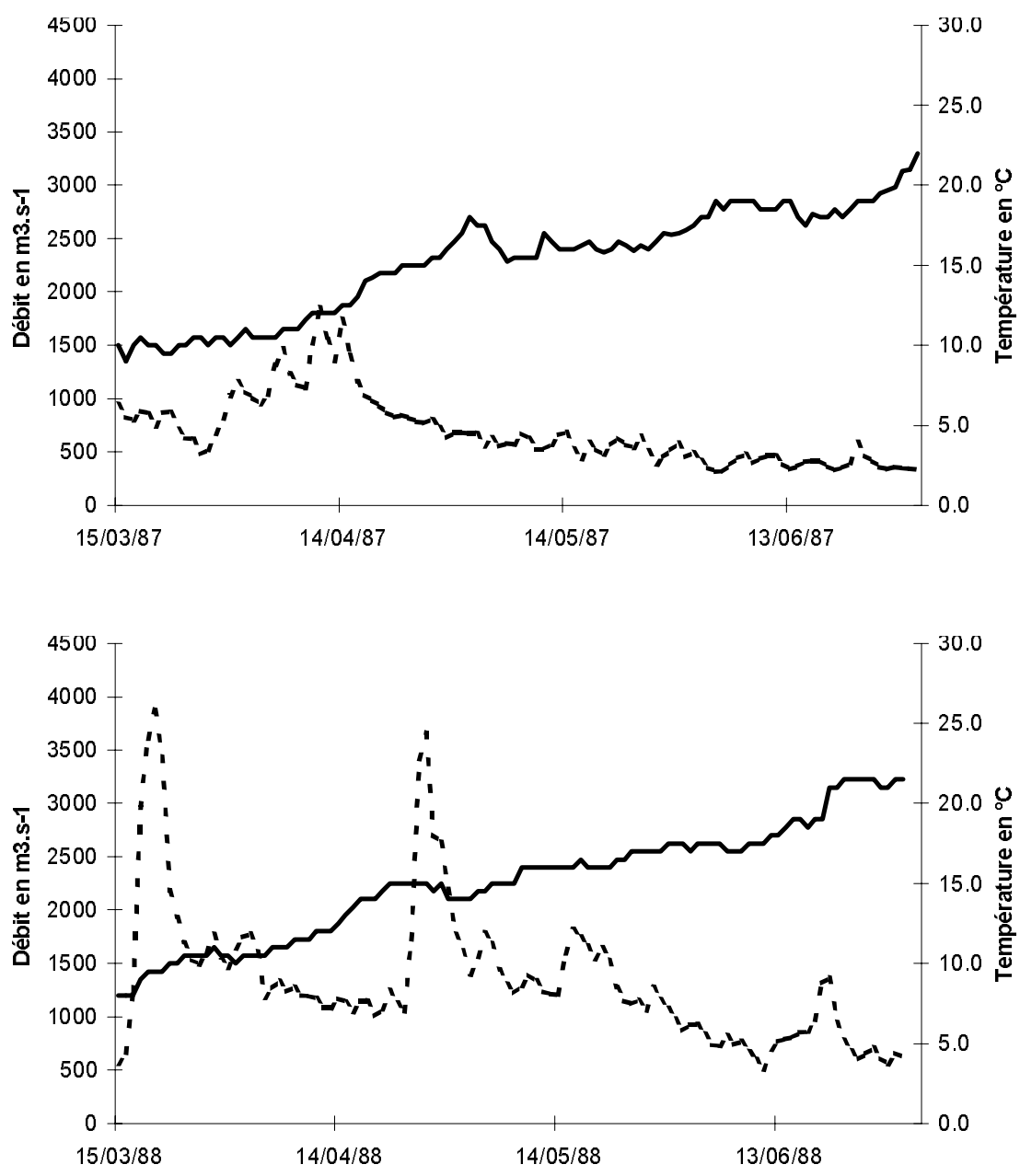

\section{Figure 3}

Chroniques de la température de l'eau en estuaire et du débit fluvial de la Garonne pour les saisons de migration 1985-1988.

\section{Figure 3}

Time series of water temperature in estuary and Garonne river flow for migration seasons 1985-1988.

L'identité de l'allure générale dans les deux secteurs permet de considérer que le laps de temps séparant les pics dans le secteur aval et dans le secteur amont (3-4 j) correspond au temps mis par les aloses pour couvrir la distance entre les centres de gravité des deux secteurs $(60-70 \mathrm{~km})$ ce qui correspond à une vitesse moyenne de 17 $23 \mathrm{~km} . \mathrm{j}^{-1}$.

\section{Rythme interne et rythme des marées}

Dans le secteur aval l'autocorrélogramme (Figure 4) fait ressortir de façon significative un rythme net d'une périodicité de 14-15 j. Pour trois des saisons analysées (1986-1988) le phénomène se répète de façon fidèle, la saison 1985, par contre, montre une périodicité de $28 \mathrm{j}$. 


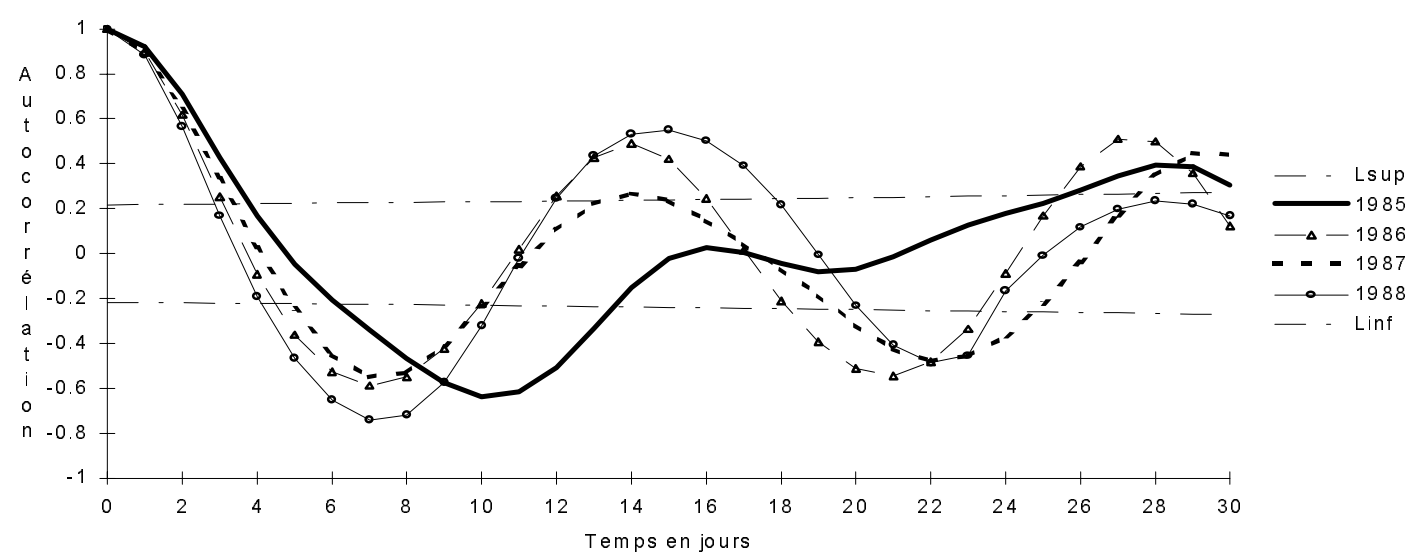

\section{Figure 4}

Autocorrélogramme de l'activité de migration dans le secteur aval (1985-1988). Les deux lignes tiretées indiquent le seuil de significativité de l'autocorrélation $(p=0,05)$.

\section{Figure 4}

Migration activity autocorrelation function in the downstream sector (1985-1988). The two doted lines indicate the significance threshold of the autocorrelation function $(p=0.05)$.

Dans le secteur amont (Figure 5) on ne retrouve pas de rythme significatif. On peut toutefois déceler pour 1986 et 1987 la trace d'un rythme d'une périodicité de 7 j. En 1985 on retrouve comme dans le secteur aval une périodicité de $28 \mathrm{j}$.

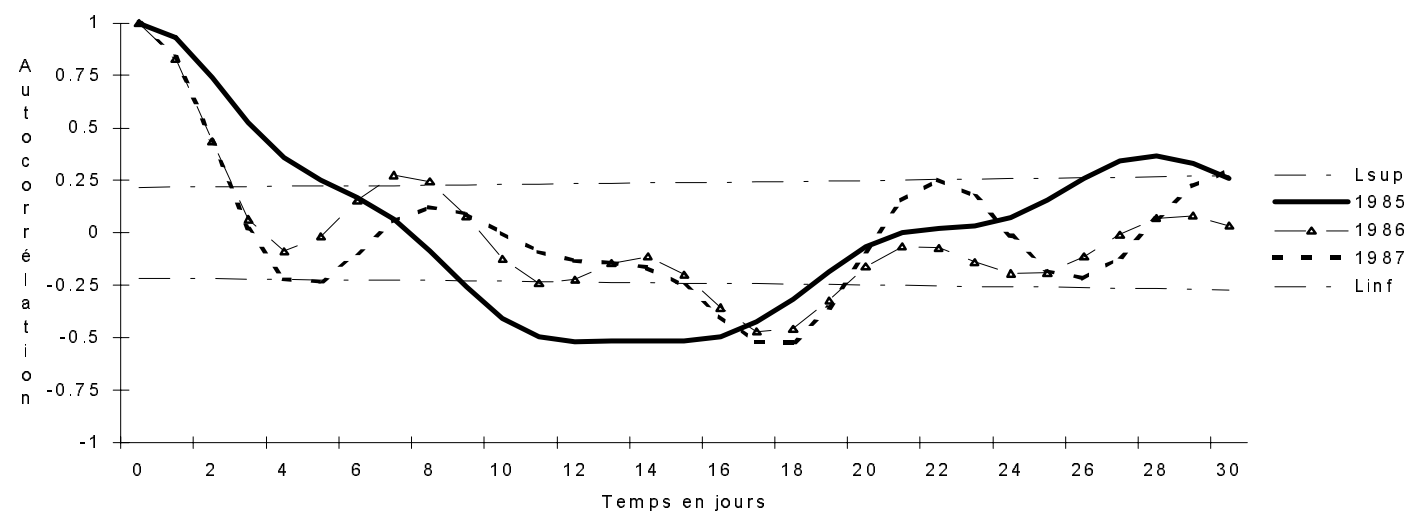

\section{Figure 5}

Autocorrélogramme de l'activité de migration dans le secteur amont (1985-1987). Les deux lignes tiretées indiquent le seuil de significativité de l'autocorrélation $(p=0,05)$.

\section{Figure 5}

Migration activity autocorrelation function in the upstream sector (1985-1987). The two doted lines indicate the significance threshold of the autocorrelation function $(p=0.05)$. 
L'existence d'un rythme d'une périodicité de 14-15 j. sur le secteur aval fait fortement penser à un lien avec les marées pour lesquelles la périodicité est d'environ $15 \mathrm{j}$. L'analyse de la corrélation croisée montre pour le secteur aval une corrélation significative (Figure 6), par contre le décalage entre le rythme des marées et la migration est variable d'une année à l'autre.

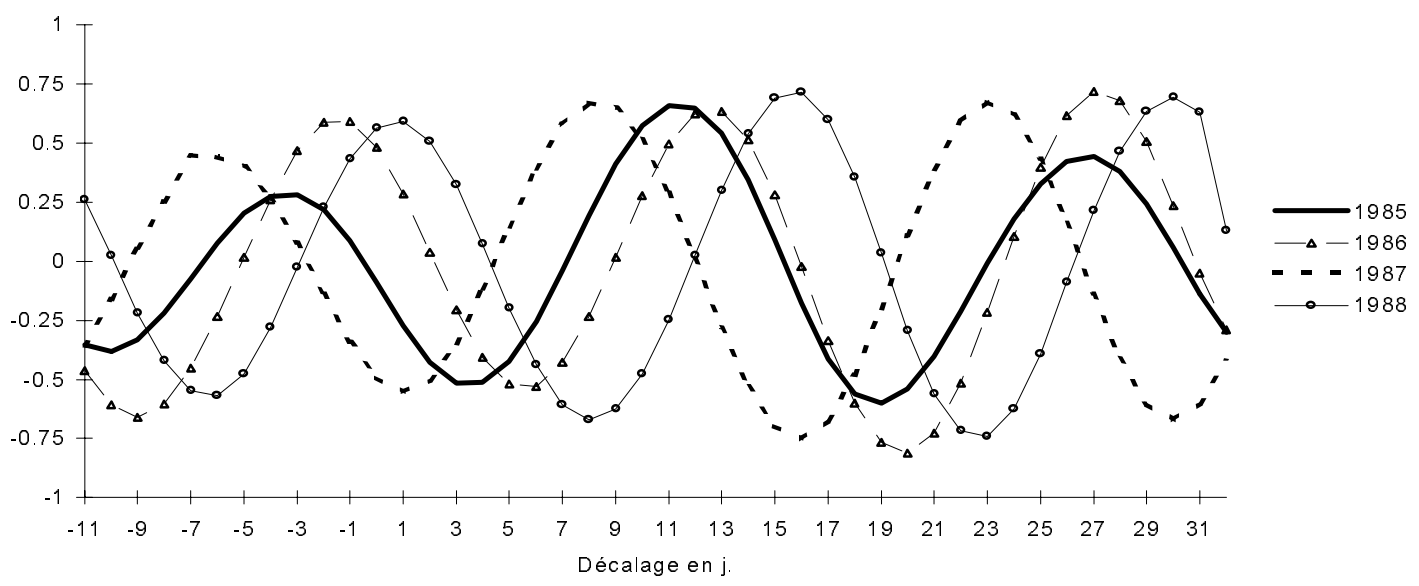

Figure 6

Corrélogramme croisé avec retard de l'activité migratoire vis à vis des coefficients de marée pour la migration en zone aval (1985-1988).

Figure 6

Migration activity crosscorrelation with tidal coefficients vs. lag for downstream sector (1985-1988).

Pour le secteur amont les corrélations sont non significatives et variables dans leur forme d'une saison à l'autre (Figure 7).

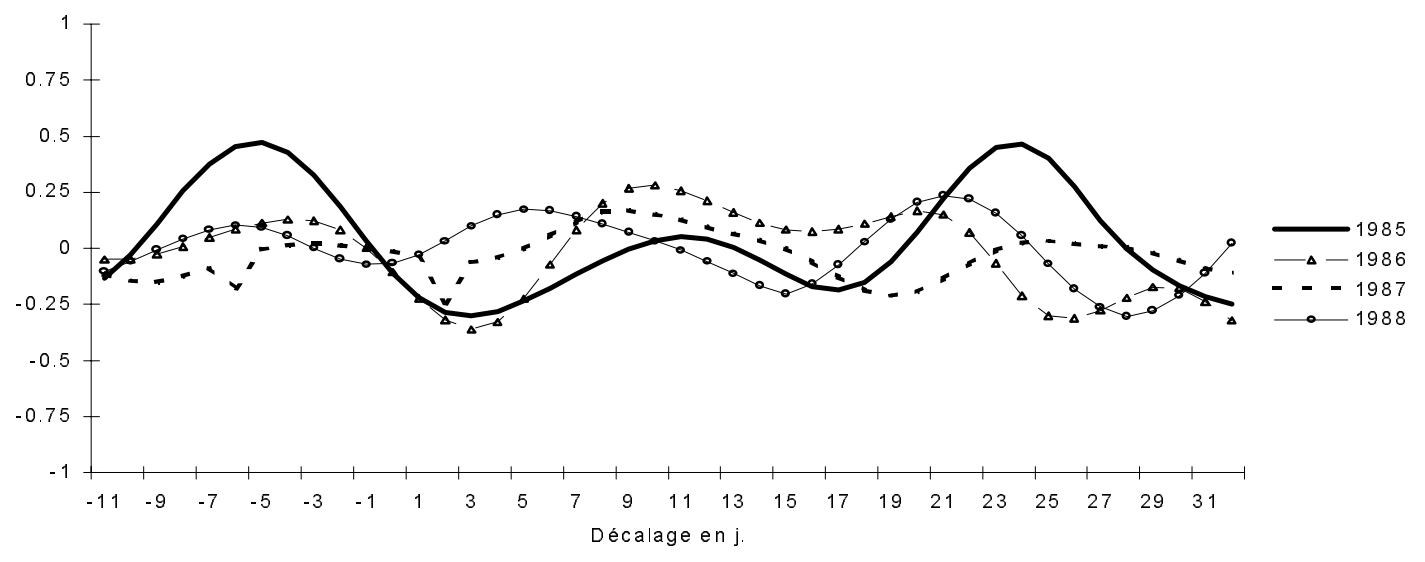

Figure 7

Corrélogramme croisé avec retard de l'activité migratoire vis à vis des coefficients de marée pour la migration en zone amont (1985-1987).

\section{Figure 7}

Migration activity crosscorrelation with tidal coefficients vs. lag for upstream sector (1985-1987). 


\section{DISCUSSION}

Pour les quatre saisons étudiées les dates de début et de fin de migration sont quasiment identiques ( $1^{\text {er }}$ avril-20 juin) seule la forme de la tendance change. II a été montré par ailleurs qu'une baisse de la température diminue l'activité migratoire des aloses, y compris en estuaire (ex. LEGGETT et WHITNEY, 1972 ; RICHKUS, 1974), si ce refroidissement est suffisamment fort et prolongé son action peut influencer la tendance de la migration. Au vu des Figures 2 et 3 on peut donc considérer que le phénomène migratoire de la grande alose est fondamentalement unimodal (une seule vague de migration). Mais en cas de baisse forte et prolongée de la température, la migration peut toutefois être retardée (comme en 1986) ou devenir bimodale (comme en 1985) et ceci même si la température demeure au dessus de la température seuil (Figures 2 et 4).

La migration des aloses dans l'estuaire de la Gironde débute au moment de l'année où la température de l'eau fluviale passe progressivement au dessus de celle des eaux côtières (C.N.EX.O, 1977), s'il survient un refroidissement on peut supposer que l'entrée des aloses dans l'estuaire est freinée voire stoppée. De la même façon au delà d'une certaine température de l'eau douce, il est possible que l'entrée soit à nouveau freinée. La migration aurait ainsi lieu dans une fenêtre thermique du même type que celle décrite par LEGGETT et WHITNEY (1972) pour lesquels $90 \%$ des captures de $A$. sapidissima sur la rivière Potomac avaient lieu entre 12 et $21^{\circ} \mathrm{C}$. L'existence d'une borne supérieure ne nous semble pas pour autant démontrée, si c'était le cas il serait nécessaire de s'interroger sur le devenir des poissons "empêchés» de rentrer dans les estuaires et sur les conséquences en terme de dynamique de population.

La température minimale pour laquelle nous avons observé des migrations lors des saisons de migration suivies est voisine de celle indiquée par MENNESSON-BOISNEAU et al. (2000) puisque seulement un faible pourcentage des migrants $(1985,8 \% ; 1986$, $8 \% ; 1987,7 \% ; 1988,1 \%)$ traverse l'estuaire à une température inférieure à $11^{\circ} \mathrm{C}$. Les crues ne s'accompagnant pas d'une baisse marquée de la température (ex. 1988) ne semblent pas avoir d'influence particulière sur la tendance de la migration, on rejoint ainsi les conclusions de DAUTREY et LARTIGUE (1983).

Les techniques de traitement du signal ont déjà été utilisées pour analyser le comportement des aloses vis à vis de phénomènes rythmiques (lune, marée, nycthémère, etc.) (SAILA et al., 1972 ; RICHKUS, 1974 ; MENNESSON-BOISNEAU, 1990 ; BOURQUE et al., 1999).

Les analyses que nous avons conduites à partir des statistiques de captures permettent de connaître certains éléments du comportement des poissons en migration. Ceci s'avère une alternative à la télémétrie acoustique pour des espèces fragiles comme $A$. alosa et $A$. fallax. Cela permet également d'établir le comportement à partir d'informations concernant une partie importante du flux de migrants (les individus capturés) et non pas seulement quelques individus.

Le corrélogramme de la migration dans le secteur aval montre un rythme très net avec toutefois un décalage variable avec les coefficients de marées. RICHKUS (1974) aboutit au même type de résultats concernant le comportement de $A$. pseudoharenus dans une rivière de la côte est des USA. Ces fluctuations cycliques, qui s'ajoutent à la tendance, peuvent être interprétées comme la résultante du comportement de l'alose dans un environnement estuarien hydrauliquement fluctuant. L'alose se dirige vers l'amont mais contrairement à des espèces avec de moins bonnes capacités natatoires comme la civelle d'anguille ou le flet, ou au contraire bonnes nageuses comme le saumon Oncorhynchus nerka (LEVY et CADENHEAD, 1995), elle ne semble pas utiliser les courants de marée de façon sélective (DODSON et al., 1972). BOURQUE et al. (1999) ont montré par simulation dans le cas de $O$. nerka que les courants de marée côtiers et 
estuariens généraient des variations de densité de même période que celle des marées sans pour autant que cela soit lié à un comportement particulier des poissons. Le même phénomène pourrait expliquer, dans le cas des aloses migrant en Gironde, l'existence d'un rythme de migration très net sur le secteur aval s'estompant au fur et à mesure de la remontée des aloses dans le bassin versant. Les décalages entre la migration et les marées, variables selon les années, pourraient être liés à la migration initiale marine ou à des modifications inter-annuelles des champs de courants côtiers ou estuariens. Ceci mériterait de faire l'objet d'une validation par simulation à l'aide d'un modèle hydraulique.

Dans le secteur amont, le décalage progressif du rythme des marées par rapport à la zone marine pourrait expliquer l'absence de rythme à une période de 14 j et la mauvaise corrélation entre la migration et les marées. Les traces de périodicité à $7 \mathrm{j}$ pourraient venir du fonctionnement pulsatile de la pêcherie. En effet, l'existence d'une relève hebdomadaire du samedi soir au dimanche soir et la reprise des activités commerciales après le repos dominical entraînent des captures généralement plus importantes le lundi que les autres jours de la semaine.

Cette absence de modification comportementale vis à vis d'un courant fluctuant de la part d'un poisson bon nageur peut paraître surprenant, mais dans leur analyse théorique concernant les stratégies de nage, TRUMP et LEGGETT (1980) concluent que, dans un environnement avec des courants variables comme dans les estuaires, il y a une dépense d'énergie minimale lorsque ces variations sont ignorées et que le poisson maintient une vitesse de nage constante. Cette stratégie permettrait aux poissons, pour le même coût énergétique, de remonter plus haut dans le bassin versant.

Notre estimation de la vitesse de migration est très voisine de celle estimée par STEINBACH et al. (1986) sur la partie fluviale de la Loire $\left(20,5 \mathrm{~km}^{-1}{ }^{-1}\right)$ et par MENNESSON-BOISNEAU (1990) pour la partie fluviale de l'estuaire de la Loire $\left(21 \mathrm{~km} . \mathrm{j}^{-1}\right)$.

\section{CONCLUSION}

La migration de la grande alose dans l'estuaire de la Gironde se déroule à la même période chaque année et en une seule vague de migration. Ce flux unimodal peut toutefois être déformé par un refroidissement à condition qu'il soit intense et prolongé, on peut alors observer, selon le moment auquel il survient, une migration bimodale. On pose l'hypothèse que les aloses en migration ont une vitesse de nage régulière et que leur passage à travers les champs de courants côtiers et estuariens créés par les marées induit, comme cela a été montré par simulation sur une autre espèce, un rythme interne de périodicité à 14 jours et de décalage variable avec les marées. La grande alose ne semble pas chercher comme d'autres espèces à adapter sa nage aux fluctuations des courants de marées. Dans le secteur amont, cette périodicité est perturbée à la fois par l'hydraulique et par le comportement de la pêcherie.

\section{REMERCIEMENTS}

Ce travail n'aurait pas été possible sans la collaboration des pêcheurs de l'estuaire de la Gironde, qu'ils en soient remerciés. Je tiens également à remercier Patrick LAMBERT et Philippe JATTEAU pour leurs remarques constructives et Christine GAZEAU pour l'aide apportée aux illustrations de cet article.

\section{BIBLIOGRAPHIE}

ALLEN G.P., 1972. Etude des processus sédimentaires dans l'estuaire de la Gironde. Thèse Doctorat ès Sciences Naturelles. Université de Bordeaux I. 314 p. 
BAGLINIÈRE J.L., ELIE P., (Eds.) 2000. Les aloses de l'Atlantique-Est et de la Méditerranée-Ouest (Alosa alosa et Alosa fallax spp.), biologie, écologie, taxinomie et influence des activités humaines. Coédition Cemagref-INRA, $314 \mathrm{p}$.

BELAUD A., LABAT R., 1985. Le comportement migratoire des aloses (Alosa alosa L.) dans le canal de restitution de l'usine de Golfech, effet de la température. Ichtyophysiolo. Acta, 9, 177-186.

BONNEFILLE R., 1970. Etude de l'aménagement de l'estuaire de la Gironde. Rapport $\mathrm{n}^{\circ}$ 9. Etude synthétique du régime hydraulique. Laboratoire national d'hydraulique, Chatou, HC042 R411, t. 690 DHM.

BOURQUE M.C., LEBLOND P.H., CUMMINS P.F., 1999. Effects of tidal currents on Pacific salmon migration : results from a fine resolution coastal model. Can. J. Fish. Aquat. Sci., 56, 839-846.

CASTELNAUD G., 1978. Etude de la pêche aux filets et aux engins dans l'estuaire de la Gironde. Thèse de Doctorat de 3ème cycle. Université de Bordeaux III. 198 p.

CASTELNAUD G., ROCHARD E., LE GAT Y., 2001. Analyse de la tendance de l'alose Alosa alosa en Gironde à partir de l'estimation d'indicateurs halieutiques sur la période 1977-1998. Bull. Fr. Pêche Piscic., 362/363, 969-995.

COX D.R., LEWIS P.A.W., 1966. The statistical analysis of series of events. Wiley and Sons Inc. London, $266 \mathrm{p}$.

C.N.EX.O., 1977. Etude écologique du Blayais. Rap. final E.D.F. 495 p.

DAUTREY R., LARTIGUE J.P., 1983. Recherches sur la migration des aloses (Alosa alosa) et des truites de mer (Salmo trutta) en Garonne (Site de Golfech). Thèse de doctorat de 3ème cycle, sciences et techniques en production animale, option ichtyologie appliquée. INP Toulouse, $212 \mathrm{p}$.

DODSON J.J., LEGGETT W.C., JONES R.A., 1972. The behavior of adult American Shad (Alosa sapidissima) during migration from salt to fresh water as observed by ultrasonic tracking techniques. J. Fish. Res. Bd. Canada, 29, 1445-1449.

FORTIER L., LEGENDRE L., CARDINAL A., TRUMP C.L., 1978. Variabilité à court terme du phytoplancton de l'estuaire du St Laurent. Mar. Biol., 46, 349-354.

KATZ H.M., 1986. Migrational swimming speeds of the American shad, Alosa sapidissima, in the Connecticut River, Massachusetts, USA. J. Fish. Biol., 29, 5 (supplement A), 189-197.

LATOUCHE C., JOUANNEAU J.M., 1994. Etude de la dynamique de l'eau et des sédiments 8-21. In : MAUVAIS J.L., GUILLAUD J.F., coord. Livre blanc de l'estuaire de la Gironde, IFREMER, Agence de l'eau Adour-Garonne, 115 p.

LEGENDRE L., LEGENDRE P., 1979. Ecologie numérique Tome 2, Le traitement multiple des données écologiques. Masson, PUQ, ed., $260 \mathrm{p}$.

LEGGETT W.C., WHITNEY R.R., 1972. Water temperature and migrations of American shad. Fish. Bull., 70 (3), 659-670.

LEVY D.A., CADENHEAD A.D., 1995. Selective tidal stream transport of adult sockeye salmon (Oncorhynchus nerka) in the Fraser river estuary. Can. J. Fish. Aquat. Sci., $52,1-12$.

MAC CLEAVE J.D., WIPPELHAUSER G.S., 1987. Behavioral aspects of selective tidal stream transport in juvenile American eels. American Fisheries Society Symposium, 1, 138-150.

MARTIN VANDEMBULCKE D., 1999. Dynamique de la population de la grande alose (Alosa alosa L. 1758) dans le bassin versant Gironde-Garonne-Dordogne (France) : analyse et prévision par modélisation. Thèse de doctorat de l'université de Toulouse. $115 \mathrm{p}$.

MASSMAN W.H., PACHECO A.L., 1957. Shad catches and water temperatures in Virginia. J. Wildl. Manage., 21 (3), 351-352.

MAURICE L., 1994. La qualité des eaux de l'estuaire. 32-45. In: MAUVAIS J.L., GUILLAUD J.F., coord. Livre blanc de l'estuaire de la Gironde, IFREMER, Agence de l'eau Adour-Garonne, $115 \mathrm{p}$. 
MENNESSON-BOISNEAU C., 1990. Migration, répartition, reproduction et caractéristiques biologiques des aloses Alosa sp. dans le bassin de la Loire. Thèse de doctorat de l'Université de Rennes I, $106 \mathrm{p}$.

MENNESSON-BOISNEAU C., APRAHAMIAN M., SABATIÉ M.R., CASSOU-LEINS J.J., 2000. Remontée migratoire des adultes. In : BAGLINIĖRE J.L., ELIE P., (Eds.). Les aloses de l'Atlantique-Est et de la Méditerranée-Ouest (Alosa alosa et Alosa fallax spp.), biologie, écologie, taxinomie et influence des activités humaines. 55-72. Coédition Cemagref-INRA.

NEVES R.J., DEPRES L., 1979. The oceanic migration of american shad, Alosa sapidissima, along the Atlantic coast. Fish. Bull., 77, 199-212.

PARKER J.A., 1992. Migratory patterns and exploitation of American shad in the nearshore ocean waters of south-eastern North Carolina. N. Am. J. Fish. Manage., 12, $752-759$

PROUZET P., MARTINET J.P., BADIA J., 1994. Caractérisation biologique et variation des captures de la grande alose (Alosa alosa) par unité d'effort sur le fleuve Adour (Pyrénées Atlantiques, France). Aquat. Living Resour., 7, p. 1-10.

RICHKUS W.A., 1974. Factors influencing the seasonal and daily patterns of alewife (Alosa pseudoharengus) migration in a Rhode Island river. J. Fish. Res. Board Can., 31, 1485-1497.

ROCHARD E., 1992. Mise au point d'une méthode de suivi de l'abondance des amphihalins dans le système fluvio-estuarien de la Gironde, application à l'étude écobiologique de l'esturgeon Acipenser sturio. Thèse de doctorat de l'université de Rennes I, $315 \mathrm{p}$.

ROULE L., 1924. Le thermotropisme dans la migration de l'alose. In : 48ème session de l'association française pour l'avancement des sciences. p. 954-957.

SABATIÉ M.R., 1993. Recherches sur l'écologie et la biologie des aloses au Maroc (Alosa alosa Linné, 1758 et Alosa fallax Lacépède, 1803). Exploitation et taxinomie des populations atlantiques; bioécologie des aloses de l'Oued Sebou. Thèse de doctorat, Université de Bretagne Occidentale, $326 \mathrm{p}$.

SAILA S.B., POLGAR T.T., SHEEHY D.J., FLOWERS J.M., 1972. Correlations between alewife activity and environmental variables at a fishway. Trans. Am. Fish. Soc., 4, 583-594.

SAURIAU P.G., ROBIN J.P., MARCHAND J., 1991. Les mortalités estivales du mulet Liza ramada en Loire : dysfonctionnement cyclique du rôle de voie de migration d'un estuaire. In : Actes du colloque « Le littoral, ses contraintes environnementales et ses conflits d'utilisation », Nantes, 115-119.

STEINBACH P., GUENEAU P., AUTUORO A., BROUSSARD D., 1986. Radio-pistage de grandes aloses adultes en Loire. Bull. Fr. Pêche Piscic., 302, 106-117.

TALBOT G., 1954. Factors associated with fluctuations in abundance of Hudson river shad. Fish. Bull., 101, 373-413.

TAVERNY C., 1991. Contribution à la connaissance de la dynamique des populations d'aloses (Alosa alosa et Alosa fallax) dans le système fluvio-estuarien de la Gironde : pêche, biologie et écologie. Etude particulière de la dévalaison et de l'impact des activités humaines. Thèse de doctorat de l'université de Bordeaux I, $451 \mathrm{p}$.

TAVERNY C., CASSOU-LEINS J.J., CASSOU-LEINS F., ELIE P., 2000. De l'œuf à l'adulte en mer. In : BAGLINIĖRE J.L., ELIE P., (Eds.). Les aloses de l'AtlantiqueEst et de la Méditerranée-Ouest (Alosa alosa et Alosa fallax spp.), biologie, écologie, taxinomie et influence des activités humaines. 93-124. Coédition Cemagref-INRA.

TAVERNY C., ELIE P., 2001. Répartition spatio-temporelle des aloses Alosa alosa (LINNÉ, 1766) et Alosa fallax (LACÉPĖDE, 1803) dans le golfe de Gascogne. Bull. Fr. Pêche Piscic., 362/363, 803-821.

TRUMP C.L., LEGGETT W.C., 1980. Optimum swimming speeds in fish : the problem of currents. Can. J. Fish. Aquat. Sci., 37, 1086-1092. 
